\title{
Minimal symmetric differences of lines in projective planes
}

\author{
Paul Balister* $\quad$ Béla Bollobás ${ }^{\dagger} \quad$ Zoltán Füredi $^{\ddagger} \quad$ John Thompson ${ }^{\S}$
}

July 20, 2021

\begin{abstract}
Let $q$ be an odd prime power and let $f(r)$ be the minimum size of the symmetric difference of $r$ lines in the Desarguesian projective plane $P G(2, q)$. We prove some results about the function $f(r)$, in particular showing that there exists a constant $C>0$ such that $f(r)=O(q)$ for $C q^{3 / 2}<r<q^{2}-C q^{3 / 2}$.
\end{abstract}

\section{Introduction}

Let $q$ be an odd prime power and consider the Desarguesian projective plane $P G(2, q)$. (For detailed definitions of lines, coordinates, conics, etc, see, e.g., the monograph Hirschfeld [11].) Write $\mathcal{P}$ and $\mathcal{L}$ for the set of points and lines of $P G(2, q)$ respectively. We shall consider the subsets of $\mathcal{P}$ or $\mathcal{L}$ as elements of a vector space isomorphic to $\mathbb{F}_{2}^{N}, N:=q^{2}+q+1$, and will switch between the 'subset' and 'vector' interpretations without further comment. For example, for subsets $A$ and $B$ of $\mathcal{P}$ or $\mathcal{L}, A+B$ represents the symmetric difference of $A$ and $B$.

Define for $0 \leq r \leq N$,

$$
f(r)=\min \left\{\left|\sum_{i=1}^{r} \ell_{i}\right|: \ell_{1}, \ldots, \ell_{r} \in \mathcal{L} \text { distinct }\right\},
$$

that is the minimal symmetric difference of $r$ lines in $P G(2, q)$.

The problem of determining $f(r)$ is motivated by the fact that it is an algebraic version of the Besicovitch-Kakeya [3] problem in a projective plane - determining the

${ }^{*}$ Department of Mathematical Sciences, University of Memphis, Memphis TN 38152, USA

${ }^{\dagger}$ Department of Pure Mathematics and Mathematical Statistics, Wilberforce Road, Cambridge CB3 0WB, UK, and Department of Mathematical Sciences, University of Memphis, Memphis TN 38152, USA

${ }^{\ddagger}$ Alfréd Rényi Institute of Mathematics, 13-15 Reáltanoda Street, 1053 Budapest, Hungary. Research supported in part by the Hungarian National Science Foundation OTKA 104343, and by the European Research Council Advanced Investigators Grant 267195.

${ }^{\S}$ Department of Pure Mathematics and Mathematical Statistics, Wilberforce Road, Cambridge CB3 OWB, UK 
minimum size of a set that contains lines (or segments) in many directions. For more results on Kakeya's problem in the finite fields see [10, 5] and the references there.

Given a set $R$ of lines in $P G(2, q)$, call a point odd if it is incident with an odd number of lines in $R$, and define the terms 'even point', 'single point', 'double point', etc., analogously. Let $\mathcal{P}^{o}(R)$ be the set of odd points, and let $\mathcal{P}^{e}(R), \mathcal{P}^{k}(R), \mathcal{P} \geq k(R)$ be defined analogously as the set of points that are even, multiplicity $k$, and multiplicity at least $k$, respectively.

Dually, for $S \subseteq \mathcal{P}$, define $\mathcal{L}^{o}(S)$ to be the set of lines $\ell \in \mathcal{L}$ such that $|\ell \cap S|$ is odd. Define $\mathcal{L}^{e}(S), \mathcal{L}^{k}(S)$, and $\mathcal{L}^{\geq k}(S)$ analogously.

By duality of lines and points in the projective plane $P G(2, q)$ we can rewrite (1) in the equivalent forms

$$
f(r)=\min _{R \subseteq \mathcal{L},|R|=r}\left|\mathcal{P}^{o}(R)\right|=\min _{S \subseteq \mathcal{P},|S|=r}\left|\mathcal{L}^{o}(S)\right| .
$$

We shall therefore often switch the viewpoint and consider sets of points which have odd intersections with few lines.

The next observation, proved below, is that $\mathcal{P}^{o}(R)$ almost determines $R$, and $\mathcal{L}^{o}(S)$ almost determines $S$. Indeed, the $N$ vectors specified by $\mathcal{L}$ span an $(N-1)$-dimensional subspace of $\mathbb{F}_{2}^{\mathcal{P}}$ and their only linear dependency is $\sum_{\ell \in \mathcal{L}} \ell=0$. This gives that $\mathcal{P}^{o}(R)=$ $\mathcal{P}^{o}\left(R^{\prime}\right)$ iff either $R=R^{\prime}$ or $R^{\prime}=\mathcal{L} \backslash R$. Indeed, it is well known that the $N \times N$ point line 0-1 incidency matrix $A$ has rank $N-1$ (one can consider $A A^{T}=J+q I$ and this has rank $N-1$ over $\mathbb{F}_{2}$, see, e.g., Ryser [14]). The following useful lemma is based on this observation.

Lemma 1. If $R=\mathcal{L}^{o}(S)$ then $|R|$ is even and either $S=\mathcal{P}^{e}(R)$ (if $|S|$ is odd) or $S=\mathcal{P}^{o}(R)$ (if $|S|$ is even). Dually, if $S=\mathcal{P}^{o}(R)$ then $|S|$ is even and either $R=\mathcal{L}^{e}(S)$ (if $|R|$ is odd) or $R=\mathcal{L}^{o}(S)$ (if $|R|$ is even).

Proof. The maps $\mathcal{L}^{o}$ and $\mathcal{P}^{o}$ can be thought of as $\mathbb{F}_{2}$-linear maps between the set of subsets of $\mathcal{P}$ and $\mathcal{L}$, each regarded as a vector space isomorphic to $\mathbb{F}_{2}^{N}$. For $p \in \mathcal{P}$, $\left|\mathcal{L}^{o}(\{p\})\right|=|\{\ell \in \mathcal{L}: p \in \ell\}|=q+1$ is even, so $\left|\mathcal{L}^{o}(S)\right|$ is even for all $S \subseteq \mathcal{P}$. Moreover

$$
\mathcal{P}^{o}\left(\mathcal{L}^{o}(\{p\})\right)=\sum_{\ell \ni p} \ell=\mathcal{P}-\{p\} \in \mathbb{F}_{2}^{\mathcal{P}}
$$

as the number $q+1$ of lines through $p$ is even and there is a unique line through $p$ and $p^{\prime}$ for every $p^{\prime} \neq p$. By linearity, $\mathcal{P}^{o}\left(\mathcal{L}^{o}(S)\right)=\sum_{p \in S}(\mathcal{P}-\{p\})=S$ when $|S|$ is even, and so $\mathcal{P}^{o}$ has rank at least $N-1$. Also, $\mathcal{P}^{o}(\mathcal{L})=\emptyset$ as every point is in an even number of lines. Hence the kernel of $\mathcal{P}^{o}$ is $\{0, \mathcal{L}\}$. Similarly the kernel of $\mathcal{L}^{o}$ is $\{0, \mathcal{P}\}$. The result now follows as $\mathcal{P}^{e}(R)=\mathcal{P} \backslash \mathcal{P}^{o}(R)$ and $\mathcal{L}^{e}(R)=\mathcal{L} \backslash \mathcal{L}^{o}(R)$.

Lemma 2. For $0 \leq r \leq N, f(N-r)=f(r)$.

Proof. Replacing any set $R=\left\{\ell_{1}, \ldots, \ell_{r}\right\}$ by its complement $\mathcal{L} \backslash R$ and noting that $\sum_{\ell \notin R} \ell=\sum_{\ell \in R} \ell$, we find that $f(N-r) \leq f(r)$. Reversing the roles of $r$ and $N-r$ gives $f(N-r) \geq f(r)$. 
Lemma 3. Let $R$ be any set of $r$ lines in $\mathcal{L}$. Then

$$
r(q+2-r) \leq\left|\mathcal{P}^{o}(R)\right| \leq r q+1
$$

and

$$
\left|\mathcal{P}^{o}(R)\right| \equiv r(q+2-r) \bmod 4 \text {. }
$$

In particular, $f(r) \geq r(q+2-r)$ and $f(r) \equiv r(q+2-r) \bmod 4$.

Proof. Each line of $R$ contains at least $q+1-(r-1)=q+2-r$ points that do not lie on any other line of $R$. Thus there are at least $r(q+2-r)$ points lying on a single line, and so in particular $\left|\mathcal{P}^{o}(R)\right| \geq r(q+2-r)$. On the other hand, one line contains $q+1$ points and the symmetric difference of two lines contains exactly $2 q$ points. Thus $\left|\mathcal{P}^{o}(R)\right| \leq r q+1$ for $r \leq 2$. For $r>2$ write $R=R^{\prime} \cup\left\{\ell, \ell^{\prime}\right\}$. Then by induction

$$
\begin{aligned}
\left|\mathcal{P}^{o}(R)\right| & =\left|\mathcal{P}^{o}\left(R^{\prime}\right)+\mathcal{P}^{o}\left(\left\{\ell, \ell^{\prime}\right\}\right)\right| \\
& \leq\left|\mathcal{P}^{o}\left(R^{\prime}\right)\right|+\left|\mathcal{P}^{o}\left(\left\{\ell, \ell^{\prime}\right\}\right)\right| \\
& \leq((r-2) q+1)+2 q=r q+1 .
\end{aligned}
$$

Now let $t_{i}=\left|\mathcal{P}^{i}(R)\right|$ be the set of points of multiplicity $i$. Then $\sum i t_{i}=r(q+1)$ is the number of points in all the lines counted with multiplicity, and $\sum i(i-1) t_{i}=r(r-1)$ is the number of intersection points between ordered pairs of lines counted with multiplicity. Subtracting gives $\sum i(2-i) t_{i}=r(q+2-r)$. But $i(2-i) \equiv 0 \bmod 4$ when $i$ is even and $i(2-i) \equiv 1 \bmod 4$ when $i$ is odd. Thus $r(q+2-r) \equiv \sum_{i \text { odd }} t_{i}=\left|\mathcal{P}^{o}(R)\right| \bmod 4$.

The function $f(r)$ is easily determined for $0 \leq r \leq q+1$ (and hence by Lemma 2 also for $N-q-1 \leq r \leq N)$.

Theorem 4. For $0 \leq r \leq q+1, f(r)=r(q+2-r)$.

Proof. Lemma 3 implies $f(r) \geq r(q+2-r)$, so it remains by (2) to construct a set $S$ of points with $|S|=r$ and $\left|\mathcal{L}^{o}(S)\right|=r(q+2-r)$.

Let $C=\left\{\left[s^{2}: s t: t^{2}\right]:[s: t] \in P G(1, q)\right\}$ be the conic $X Z=Y^{2}$. We note that all lines $\ell$ intersect $C$ in at most 2 points, and $|\ell \cap C|=1$ if and only if $\ell$ is one of the $q+1$ tangent lines to $C$.

Let $S$ be any subset of $C$ of size $r$. No line intersects $S$ in more than two points and so for any $p \in S$ exactly $r-1$ lines through $p$ meet $C$ at another point of $S$, while $(q+1)-(r-1)=q+2-r$ lines through $p$ fail to meet $C$ at any other point of $S$. Thus there are exactly $r(q+2-r)$ lines that meet $S$ in an odd number of points and so $\left|\mathcal{L}^{o}(S)\right|=r(q+2-r)$ as required.

The function $f(r)$ cannot vary too rapidly; trivially we have $|f(r+1)-f(r)| \leq q+1$. In fact, we can say slightly more.

Theorem 5. For $0<r<N-2,|f(r+1)-f(r)| \leq q-1$.

Note that $f(0)=f(N)=0$ and $f(1)=f(N-1)=q+1$, so this result fails for $r=0, N-1$. On the other hand, the inequality can be sharp. For example, $f(2)-f(1)=f(q+1)-f(q)=q-1$ by Theorem 4 . There are other examples, e.g., $f(2 q-1)=q+1$ and $f(2 q)=2$ (see Theorem 13 below). 
Proof. Assume $|R|=r$ and $\mathcal{P}^{o}(R)=S$ with $|S|=f(r)$. Note that $S \neq \emptyset$ as $R \neq \emptyset, \mathcal{L}$. Pick $p \in S$. Assume every line $\ell$ through $p$ intersects $S$ in an odd number of points. Then every line through $p$ intersects $S \backslash p$ is an even number of points. Since distinct lines through $p$ partition $S \backslash p$, we see that $|S \backslash p|$ is even and hence $|S|$ is odd, contradicting Lemma 1. Thus there exists a line $\ell_{e}$ that meets $S$ in an even (and positive) number of points. If all $\ell \in \mathcal{L}$ met $S$ in an even number of points then $\mathcal{L}^{\circ}(S)=\emptyset$ and so $S=\emptyset$ or $\mathcal{P}$, a contradiction. Thus there exists a line $\ell_{o}$ that meets $S$ in an odd number of points. As $R=\mathcal{L}^{o}(S)$ or $\mathcal{L}^{e}(S)$, either $\ell_{e}$ or $\ell_{o}$ fails to lie in $R$. Adding such a line to $R$ increases $r$ by one and increases $S$ by at most $q-1$, implying $f(r+1)-f(r) \leq q-1$.

Replacing $r$ by $N-r-1$ and applying Lemma 2 gives $f(r+1)-f(r)=-(f(N-$ $r)-f(N-r-1)) \geq-(q-1)$, completing the proof of Theorem 5 .

\section{The case of $q+2$ lines}

Our next aim is to prove that the jump $f(q+2)-f(q+1)=f(q+2)-(q+1)$ is not too small.

Theorem 6. $f(q+2)=2 q-2$ for $q \leq 13$. More generally, for $q \geq 7$ we have $\frac{3}{2}(q+1) \leq f(q+2) \leq 2 q-2$.

To prove this we shall use several lemmas, some classical results of this topic. Most of their proofs use either Rédei's method (see. e.g., [13]) or some version of Combinatorial Nullstellensatz (see, e.g., [1, Theorem 1.2]). Arrangements of $q+2$ lines are the most investigated part of finite geometries. In the following, a triple point with respect to a set of lines $R$ will refer to a point which lies on at least three lines.

Lemma 7 (Bichara and Korchmáros [2]). Let $R$ be a set of $q+2$ lines in $P G(2, q)$. Then there are at most two lines without triple points.

A blocking set in the affine plane $A G(2, q)$ or in the projective plane $P G(2, q)$ is a set $B$ of points such that each line is incident with at least one point of $B$.

Lemma 8 (Brouwer and Schrijver [6] and Jamison [12]). Let $B$ be a blocking set in $A G(2, q)$. Then $B$ consists of at least $2 q-1$ points.

Lemma 9 (Szőnyi [15]). Let $B$ be a minimal blocking set in $P G(2, q)$ of size less than $3(q+1) / 2$ where $q=p^{h}$ for some prime $p$. Then all lines meet $B$ in $1 \bmod p$ points.

The following lemma is contained in [5] (top of page 211) as a part of a more complex argument. For completeness we reproduce its proof here.

Lemma 10 (Blokhuis and Mazzocca [5]). Let $R$ be a set of $q+2$ lines with at least one of the lines containing no triple points. Then the number of odd points is at least $2 q$ minus the number of lines in $R$ without triple points.

Proof. Without loss of generality, we may assume that $R$ contains the line at infinity and that this line has no triple point. Let $L$ be the set of $q+1$ lines in $A G(2, q)$ obtained 
by restricting the remaining lines of $R$ to $A G(2, q)$. As the line at infinity contains no triple point, no two lines in $L$ are parallel. Then as $|L|=q+1$, every line $\ell$ in $A G(2, q)$ is parallel to precisely one line of $L$.

Claim. In $A G(2, q)$ the odd points block all lines in $A G(2, q)$, except those in $L$ that have no triple points.

Indeed, assume first that $\ell \notin L$. Then $\ell$ intersects $q$ of the lines in $L$; indeed it intersects all but the unique line in $L$ parallel to $\ell$. Since $q$ is odd, $\ell$ has an odd point.

Now assume $\ell \in L$ and has a triple point. As there are $q$ points in $L$ and only $q$ other lines in $L$, the fact that some point in $\ell$ meets at least two of these lines implies that there is a point of $\ell$ which meets no other line of $L$. Such a point is a single (and hence odd) point.

Adding one point from each line without a triple point (except the line at infinity) we obtain a blocking set of the affine plane, which by Lemma 8 contains at least $2 q-1$ points. The result follows.

Proof of the lower bound in Theorem [6. Let $R$ be a set of $q+2$ lines with $f(q+2)=$ $\left|\mathcal{P}^{o}(R)\right|, S:=\mathcal{P}^{o}(R)$, and let $T_{3}$ be the set of triple points. We will show that $|S| \geq$ $3(q+1) / 2$.

First, suppose that $R$ has a line without a triple point. Then by Lemmas 7 and 10 there are at least $2 q-2$ odd points.

Second, suppose all $q+2$ lines in $R$ have triple points and $|S|<2 q-2$. Since $f(q+2) \equiv 0 \bmod 4$ by Lemma 3 we may suppose that $|S| \leq 2 q-6$.

Claim. $S$ is a minimal blocking set in $P G(2, q)$.

Indeed, every line $\ell$ in $P G(2, q)$ is either in our set (in which case it contains a single point), or intersects all $q+2$ lines of $R$. As $q+2$ is odd, $\ell$ must contain an odd point.

That $S$ is minimal can be seen as follows: Let $v \in S$ and suppose on the contrary that $S \backslash\{v\}$ meets all lines. Since $v$ is an odd point, there are $2 m+1$ lines of $R$ containing it. Each of these lines contains at least $2 m-1$ additional odd (single) points of $S$. Moreover, every line $\ell$ not in $R$ has an odd number of odd points. Then if $\ell \notin R$ is a line through $v$, we have $|S \cap \ell| \geq 2$ and hence $|S \cap \ell| \geq 3$. In total we find at least $(2 m+1)(2 m-1)+2(q-2 m) \geq 2 q-1$ odd points beside $v$. This contradiction completes the proof of the Claim.

We count multiplicities of intersections as in the proof of Lemma 3, If we let $t_{i}$ be the number of points that occur in exactly $i$ of our lines, then $\sum_{i} i t_{i}=\sum_{i} i(i-1) t_{i}=$ $(q+2)(q+1)$. Thus $\sum_{i} i(i-2) t_{i}=0$, rearranging

$$
|S|=\sum_{i \text { odd }} t_{i}=\sum_{i \geq 3}(i(i-2)+(i \bmod 2)) t_{i}=4 t_{3}+8 t_{4}+16 t_{5}+24 t_{6}+\ldots
$$

Let $R_{3} \subseteq R$ be the set of lines having a single triple point, and that point has degree three, and let $R_{4} \subseteq R$ be the set of lines having a single triple point, and that point has degree at least four. Every line in $R$ has at least one triple point, the members of $R \backslash\left(R_{3} \cup R_{4}\right)$ have at least two. So adding up the degrees of triple points we obtain $\sum_{i \geq 3} i t_{i}=\sum_{\ell \in R}\left|\ell \cap T_{3}\right| \geq 2|R|-\left|R_{3}\right|-\left|R_{4}\right|$. Consider $\sum_{i \geq 4} i t_{i}$, it is an upper bound for $\left|R_{4}\right|$. Summarizing we obtain

$$
3 t_{3}+\sum_{i \geq 4} 2 i t_{i} \geq 2|R|-\left|R_{3}\right|
$$


This and (3) yield $|S| \geq 2 q+4-\left|R_{3}\right|$. Every $R_{3}$ line meets $S$ in two elements, so actually $R_{3}=\emptyset$ by Lemma 9 for $|S|<3(q+1) / 2$. This contradiction completes the proof of $|S| \geq 3(q+1) / 2$. For $q \leq 13$ we note that $3(q+1) / 2>2 q-6$, so $f(q+2)=2 q-2$.

Finally, to show $f(q+2) \leq 2 q-2$ recall that $f(q+2) \leq f(q+1)+(q-1)=2 q$ by Theorems 5 and 4 , while $f(q+2) \equiv 0 \bmod 4$ by Lemma 3 . Thus $f(q+2) \leq 2 q-2$.

This upper bound on $f(q+2)$ can also be seen in the following way. There is an action of $S L(2, q)$ on $P G(2, q)$ in which the orbits are $A, B$, and $C$, where $C$ is the conic described above, $A$ is the set of points which lie on no tangent of $C$ and $B$ is the set of points that lie on two tangents of $C$. Now $\left|\mathcal{L}^{o}(C)\right|=q+1$, so if $p \in A$ then $\left|\mathcal{L}^{o}(C \cup\{p\})\right|=(q+1)+(q+1)$ as all lines through $p$ change from having an even intersection with $C$ to having an odd intersection with $C \cup\{p\}$. On the other hand, if $p \in B$ then $\left|\mathcal{L}^{o}(C \cup\{p\})\right|=(q+1)+(q-1)-2=2 q-2$ as there are $q-1$ lines thorough $p$ with an even intersection with $C$ and an odd intersection with $C \cup\{p\}$, while there are 2 lines through $p$ that are tangent to $C$ and so have odd intersection with $C$ and even intersection with $C \cup\{p\}$. The result now follows from (2).

We conjecture that in fact the upper bound is correct in Theorem 6.

Conjecture 11. $f(q+2)=2 q-2$.

\section{Exact values near $2 q$}

A few more values of $f(r)$ are known when $r$ is small. To derive these we shall make use of the following result.

Lemma 12. For even $s, f(s)$ is the minimum even $r$ such that there exists a set $R$ of lines with $|R|=r$ and $\left|\mathcal{P}^{o}(R)\right|=s$.

Proof. Assume $R$ is a set of lines with $|R|=r$ and $\sum_{\ell \in R} \ell=S$ with $|S|=s$. Now $\left|\mathcal{L}^{o}(S)\right|$ is even while $\left|\mathcal{L}^{e}(S)\right|$ is odd. Hence $R=\mathcal{L}^{o}(S)$ as $r$ is even. Thus, by (2), $f(s) \leq r$. Conversely, if $f(s)=r$ and $|S|=s$ with $\left|\mathcal{L}^{o}(S)\right|=r$, then $r$ is even and, setting $R=\mathcal{L}^{o}(S)$, we have $|R|=r$ and $\left|\mathcal{P}^{o}(R)\right|=|S|=s$ as $s$ is even.

Theorem 13. $f(2 q-1)=q+1, f(2 q)=2, f(2 q+1)=q-1$.

Proof. If $|R|=2$ then $\left|\mathcal{P}^{o}(R)\right|=2 q$, so $f(2 q) \leq 2$ by Lemma 12. However $f(r)>0$ and $f(r)$ is even for $0<r<N$, so $f(2 q)=2$. Thus $f(2 q-1), f(2 q+1) \leq q+1$ by Theorem 5 . Also $f(2 q+1) \equiv(2 q+1)(-q+1) \equiv q-1 \bmod 4$ and $f(2 q-1) \equiv(2 q-1)(-q+3) \equiv$ $q+1 \bmod 4$ by Lemma 3. Thus it is sufficient to show that $f(2 q \pm 1)>q-3$. As $2 q \pm 1$ is odd, there exists a $R$ with $|R|=f(2 q \pm 1)$ and $\left|\mathcal{P}^{o}(R)\right|=N-(2 q \pm 1) \geq q^{2}-q$. But $\left|\mathcal{P}^{o}(R)\right| \leq q|R|+1$ by Lemma 3, so $|R|>q-3$.

\section{A graph clique decomposition lemma}

The values of $f(r)$ for $q+2<r<2 q-1$ remain to be determined, and indeed $f(r)$ is unknown for many values of $r<C q^{3 / 2}$, although some non-trivial bounds are given by 
Lemmas 19 and 20 below. For larger $r$, between $C q^{3 / 2}$ and $N-C q^{3 / 2}$, we shall show much more. Indeed it seems that $f(r)$ can be determined for most values of $r$ in this range, although an explicit description of these values seems difficult.

Suppose that $s$ is even (the case when $s$ is odd follows by considering $f(N-s)$ ). By Lemma 12 and duality it is enough to determine for each even $r$ in turn whether or not there exists a set $S$ of points such that $\left|\mathcal{L}^{o}(S)\right|=s$. Any set of points $S$ induces an edge-decomposition of the complete graph $K_{S}$ with vertex set $S$ into cliques on the sets $\ell \cap \mathcal{S}, \ell \in \mathcal{L}$. Indeed, every pair of points of $S$ lie in a unique line $\ell \in \mathcal{L}$ so each edge $K_{S}$ lies in a unique clique $K_{\ell \cap S}$. We show that $s=\left|\mathcal{L}^{o}(S)\right|$ can be determined in terms of the sizes of these cliques.

Lemma 14. Suppose $r=|S|$ is even and $\left|\mathcal{L}^{o}(S)\right|=r q-4 t$. For $\ell \in \mathcal{L}$ write $r_{\ell}=|S \cap \ell|$. Then $\sum_{\ell \in \mathcal{L}}\left\lfloor\frac{r_{\ell}}{2}\right\rfloor=\frac{r}{2}+2 t$.

Proof. As there are $q+1$ lines through each point of $S, \sum_{\ell \in \mathcal{L}} r_{\ell}=r(q+1)$. Thus

$$
r q-4 t=\left|\mathcal{L}^{o}(S)\right|=\sum_{r_{\ell} \text { odd }} 1=\sum_{\ell}\left(r_{\ell}-2\left\lfloor\frac{r_{\ell}}{2}\right\rfloor\right)=r q+r-2 \sum_{\ell}\left\lfloor\frac{r_{\ell}}{2}\right\rfloor .
$$

Hence $\sum\left\lfloor\frac{r_{\ell}}{2}\right\rfloor=\frac{r}{2}+2 t$.

Note that by Lemma $3 s=\left|\mathcal{L}^{o}(S)\right|$ must be of the form $r q-4 t$ with $0 \leq t \leq\left(\begin{array}{l}r \\ 2\end{array}\right)$. Since we are interested in the smallest $r$ for which a suitable set $S$ exists, typically we expect $t$ to be relatively small and $r$ not much bigger that $s / q$. We can therefore reduce the problem to the question of (a) whether there is any clique decomposition of $K_{r}$ into cliques of size $r_{1}, \ldots, r_{n}$ with a given value of $\sum\left\lfloor\frac{r_{i}}{2}\right\rfloor$, and (b) whether such a decomposition can be realized by a set of points inside $P G(2, q)$.

We call an edge-decomposition $\Pi$ of $K_{r}$ into cliques of orders $r_{1}, \ldots, r_{n}$ a simple decomposition if there is at most one value of $i$ with $r_{i}>3$. In other words, $K_{r}$ is decomposed as single edges, triangles, and at most one larger clique. We write $M(\Pi)$ for the sum $\sum_{i=1}^{n}\left\lfloor\frac{r_{i}}{2}\right\rfloor$.

Lemma 15. Suppose we are given an edge-decomposition $\Pi$ of $K_{r}$ with $M(\Pi)<$ $\frac{1}{4} r(\sqrt{4 r-3}-1)$. Then there exists a simple edge-decomposition $\Pi^{\prime}$ of $K_{r}$ with $M\left(\Pi^{\prime}\right)=$ $M(\Pi)$.

Proof. Assume $\Pi$ decomposes $K_{r}$ into cliques of orders $r_{1}, \ldots, r_{n}$ with $r_{1} \geq r_{2} \geq \cdots \geq r_{n}$. Let $C_{i}$ be the $i$ 'th clique. Then there are $r_{1}\left(r-r_{1}\right)$ edges from $V\left(C_{1}\right)$ to $V\left(K_{r}\right) \backslash V\left(C_{1}\right)$. Moreover, each clique $C_{i}, i>1$, can meet $C_{1}$ in at most one vertex and hence covers at most $r_{i}-1$ of these edges. Thus $\sum_{i>1}\left(r_{i}-1\right) \geq r_{1}\left(r-r_{1}\right)$ and hence

$$
M(\Pi) \geq \sum_{i=1}^{n} \frac{r_{i}-1}{2} \geq \frac{r_{1}-1}{2}+\frac{r_{1}\left(r-r_{1}\right)}{2} .
$$

On the other hand there are $\left(\begin{array}{l}r \\ 2\end{array}\right)$ edges to be covered in total, so

$$
M(\Pi) \geq \sum_{i=1}^{n} \frac{r_{i}-1}{2}=\sum_{i=1}^{n} \frac{1}{r_{i}}\left(\begin{array}{c}
r_{i} \\
2
\end{array}\right) \geq \frac{1}{r_{1}}\left(\begin{array}{l}
r \\
2
\end{array}\right) .
$$


For $r_{1}<r / 2$, the bound in (4) is increasing and the bound in (5) is decreasing as $r_{1}$ increases, so the smallest bound on $M(\Pi)$ occurs when the two bounds are equal. It can be checked that this occurs when $r=r_{1}^{2}-r_{1}+1$ with a common bound $M(\Pi) \geq$ $\frac{1}{2} r\left(r_{1}-1\right)=\frac{1}{4} r(\sqrt{4 r-3}-1)$. This contradicts the assumption on $M(\Pi)$, so we may assume $r_{1} \geq r / 2$.

Let $E_{1}$ be the set of $r_{1}\left(r-r_{1}\right)$ edges joining $C_{1}$ to the rest of $K_{r}$ and $E_{2}$ be the set of $\left(\begin{array}{c}r-r_{1} \\ 2\end{array}\right)$ edges of $K_{r}$ not meeting $C_{1}$. For each clique $C_{i}, i>1$, we note that for all $r_{i} \geq 2$,

$$
\left|E_{1} \cap E\left(C_{i}\right)\right|-\left|E_{2} \cap E\left(C_{i}\right)\right| \leq\left\lfloor\frac{r_{i}}{2}\right\rfloor \leq\left|E_{1} \cap E\left(C_{i}\right)\right|+\left|E_{2} \cap E\left(C_{i}\right)\right| .
$$

Indeed, the right hand side is just $\left(\begin{array}{c}r_{i} \\ 2\end{array}\right)$, while the left hand side is either $\left(r_{i}-1\right)-\left(\begin{array}{c}r_{i}-1 \\ 2\end{array}\right)$ or $-\left(\begin{array}{c}r_{i} \\ 2\end{array}\right)$ depending on whether or not $C_{i}$ meets some vertex of $C_{1}$. Note that the lower bound is achieved if $r_{i} \in\{2,3\}$ and $C_{i}$ meets $C_{1}$. Summing over all cliques gives

$$
\left\lfloor\frac{r_{1}}{2}\right\rfloor+\left|E_{1}\right|-\left|E_{2}\right| \leq M(\Pi) \leq\left\lfloor\frac{r_{1}}{2}\right\rfloor+\left|E_{1}\right|+\left|E_{2}\right| \text {. }
$$

Also note that $\left\lfloor\frac{r_{i}}{2}\right\rfloor \equiv\left(\begin{array}{c}r_{i} \\ 2\end{array}\right) \bmod 2$, so that $M(\Pi)$ is equivalent to either bound modulo 2 .

As $r_{1} \geq r / 2$, the graph on $E_{1} \cup E_{2}$ can be packed with $\left|E_{2}\right|$ triangles each meeting $C_{1}$. Indeed, it is enough to decompose $K_{r-r_{1}}$ completely into at most $r_{1}$ partial matchings $M_{1}, \ldots, M_{r_{1}}$ and then join each matching to a distinct vertex of $C_{1}$ to obtain sets of edge-disjoint triangles. For even $r-r_{1}$, it is well-known that $K_{r-r_{1}}$ can be decomposed into $r-r_{1}-1<r_{1}$ perfect matchings. For odd $r-r_{1}$ decompose $K_{r-r_{1}+1}$ into $r-r_{1} \leq r_{1}$ perfect matchings and remove a single vertex to give a decomposition of $K_{r-r_{1}}$ into $r-r_{1}$ partial matchings. Completing the packing of $E_{1} \cup E_{2}$ by including $K_{2}$ s covering the remaining edges of $E_{1}$ gives a decomposition $\Pi^{\prime \prime}$ of $K_{r}$ which achieves the lower bound $M_{0}=\left\lfloor r_{1} / 2\right\rfloor+\left|E_{1}\right|-\left|E_{2}\right|$ in (6). Now replacing $\left(M(\Pi)-M_{0}\right) / 2 \leq\left|E_{2}\right|$ of the triangles of this packing with three $K_{2} \mathrm{~s}$, allows us to increase $M\left(\Pi^{\prime \prime}\right)$ in steps of 2 until we get to a packing $\Pi^{\prime}$ of $C_{1}$, edges, and triangles, with $M\left(\Pi^{\prime}\right)=M(\Pi)$.

Lemma 16. Let $m=\lceil\sqrt{r-3}\rceil-1$. Then for any integer $s$ with $s \leq\left(\begin{array}{l}r \\ 2\end{array}\right), s \equiv\left(\begin{array}{l}r \\ 2\end{array}\right) \bmod 2$, and $s \geq\left\lfloor\frac{r-m}{2}\right\rfloor+\frac{m}{2}(2 r-3 m+1)$ there exists a simple decomposition $\Pi$ of $K_{r}$ with $M(\Pi)=s$.

Proof. From the proof of Lemma 15 we know that we can construct a simple a decomposition for any $s \equiv\left(\begin{array}{l}r \\ 2\end{array}\right)$ and

$$
\left\lfloor\frac{r_{1}}{2}\right\rfloor+r_{1}\left(r-r_{1}\right)-\left(\begin{array}{c}
r-r_{1} \\
2
\end{array}\right) \leq s \leq\left\lfloor\frac{r_{1}}{2}\right\rfloor+r_{1}\left(r-r_{1}\right)+\left(\begin{array}{c}
r-r_{1} \\
2
\end{array}\right)
$$

with $r_{1} \geq \frac{r}{2}$. It is a simple but tedious exercise to show that the intervals for $r_{1}=$ $\left\lceil\frac{r}{2}\right\rceil, \ldots, r-m$ cover every $s \equiv\left(\begin{array}{l}r \\ 2\end{array}\right)$ in the range from $\left\lfloor\frac{r-m}{2}\right\rfloor+\frac{m}{2}(2 r-3 m+1)$ to $\frac{3}{4}\left(\begin{array}{c}r \\ 2\end{array}\right)$. For $s>\frac{3}{4}\left(\begin{array}{l}r \\ 2\end{array}\right)$ it is enough to show that one can pack $\left(\left(\begin{array}{c}r \\ 2\end{array}\right)-s\right) / 2 \leq\left(\begin{array}{c}\lfloor r / 2\rfloor \\ 2\end{array}\right)$ triangles into $K_{r}$. This also follows from the proof of Lemma 15 where it was shown that one can pack $\left(\begin{array}{c}\lfloor r / 2\rfloor \\ 2\end{array}\right)$ triangles into $K_{r} \backslash E\left(K_{\lceil r / 2\rceil}\right)$.

Lemmas 15 and [16] show that if there exists a decomposition with $M(\Pi)=s$ then there exists a simple decomposition with $M(\Pi)=s$ except possibly in the range between about $\frac{1}{2} r^{3 / 2}$ and about $r^{3 / 2}$. There can exist non-simple decompositions in this range 
for which there is no simple decomposition. For example, the lines of a projective plane of order $q^{\prime}, q^{\prime}$ odd, give rise to a decomposition $\Pi$ of $K_{r}$ when $r=q^{2}+q^{\prime}+1$ with $M(\Pi)=\left(q^{\prime 2}+q^{\prime}+1\right)\left(q^{\prime}+1\right) / 2$ (exactly the bound in Lemma 15). One can check that for a simple decomposition to have the same value of $M(\Pi)$ would require $\frac{q^{\prime}-1}{2}<r_{1}<\frac{q^{\prime}+1}{2}$ for large $q^{\prime}$, an impossibility, so no corresponding simple decomposition exists.

\section{Realizing clique decompositions of the projective plane}

We now turn to the question of whether a simple decomposition can be realized by a set of points in $P G(2, q)$. One needs a set $S$ formed by taking a large number $r_{1}$ of points in one line, and the remaining points only on lines intersecting $S$ in at most 3 points. The proof of the following lemma provides a construction which realizes this in most relevant cases.

Lemma 17. Fix $r, 0 \leq r \leq q+1$ and assume $r_{1} \geq \max \left\{\frac{1}{3}(2 r-3),(2 r-3)-(q+1)\right\}$. Then any simple decomposition $\Pi$ of $K_{r}$ with maximal clique of order $r_{1}$ can be realized by a set of points in $P G(2, q)$.

Proof. Consider sets of points that are subsets of $C \cup L$, where $C=\left\{X Z=Y^{2}\right\}$ is the conic used in the proof of Theorem 4 and $L=\{X=d Z\}$ is a line that does not intersect $C$ (so $d$ is chosen to be a quadratic non-residue in the field $\mathbb{F}_{q}$ ). A simple calculation shows that the secant line joining $\left[s^{2}: s t: t^{2}\right]$ and $\left[s^{2}: s^{\prime} t^{\prime}: t^{\prime 2}\right]$ on $C$ meets $L$ at the point $\left[d\left(s t^{\prime}+s^{\prime} t\right): d t t^{\prime}+s s^{\prime}: s t^{\prime}+s^{\prime} t\right]$ on $L$. This mapping of pairs of points on $C$ to $L$ is more easily described by introducing the norm group $G=\mathbb{F}_{q^{2}}^{\times} / \mathbb{F}_{q}^{\times}$. The points $p=\left[s^{2}: s t: t^{2}\right] \in C$ correspond to the coset $\phi(p)=(s+t \sqrt{d}) \mathbb{F}_{q}^{\times}$and the coset $\alpha=(a+b \sqrt{d}) \mathbb{F}_{q}^{\times}$corresponds to the point $\psi(\alpha)=[d b: a: b] \in L$. The secant line through $p, p^{\prime} \in C$ then meets $L$ at $\psi\left(\phi(p) \phi\left(p^{\prime}\right)\right)$. The key point is that $G$ is cyclic of order $q+1$. Hence by taking a subset $P=\left\{p_{1}, p_{2}, \ldots, p_{s}\right\}$ of $C$ with $2 s-3 \leq q+1$ such that $\phi\left(p_{i}\right)$ form a suitable geometric progression, the secants through these points meet $L$ in only $2 s-3$ points (assuming $s \geq 2$ ). Indeed, we can take $\phi\left(p_{i}\right)=\alpha^{i}$ where $\alpha$ is a generator of $G$ so that the secants meet $L$ at the points $\psi\left(\alpha^{3}\right), \psi\left(\alpha^{4}\right), \ldots, \psi\left(\alpha^{2 s-1}\right)$. Moreover there are 4 points $\left(\psi\left(\alpha^{3}\right), \psi\left(\alpha^{4}\right), \psi\left(\alpha^{2 s-2}\right), \psi\left(\alpha^{2 s-1}\right)\right)$ on $L$ which meet just one secant, 4 which meet exactly 2 secants, etc., with 1 or 3 points meeting $\lfloor s / 2\rfloor$ secants (depending on the parity of $s$ ). Now let $P^{\prime}=\left\{p_{1}^{\prime}, \ldots, p_{t}^{\prime}\right\}$ be a set of $t$ points on the line $L$ and suppose there are $k$ secants through two points of $P$ meeting $P^{\prime}$. then $P \cup P^{\prime}$ induces a simple edge decomposition of $K_{P \cup P^{\prime}}$ with one clique of order $\left|P^{\prime}\right|$ and $k$ triangles, the remaining cliques being single edges.

We now consider the conditions on the parameter that allow us to vary $k$ between the minimum of zero and the maximum of $\left(\begin{array}{l}s \\ 2\end{array}\right)$, where $s \geq 2$. To achieve $k=0$ requires $t \leq(q+1)-(2 s-3)$ as $P^{\prime}$ must avoid all the secant lines through $P$. To achieve $k=\left(\begin{array}{l}s \\ 2\end{array}\right)$ requires $t \geq 2 s-3$ as $P^{\prime}$ must meet all secants through $P$. All values of $k$ between the minimum and maximum can be achieved one step at a time by moving some point of $P^{\prime}$ so that it meets one more secant line. Now $s=r-r_{1}$ and $t=r_{1}$ so these conditions 
become

$$
r_{1} \leq q+1-\left(2 r-2 r_{1}-3\right) \quad \text { and } \quad r_{1} \geq 2 r-2 r_{1}-3,
$$

or equivalently $r_{1} \geq(2 r-3)-(q+1)$ and $r_{1} \geq \frac{1}{3}(2 r-3)$. For $s<2$ there are no secant lines and the only restriction is $t=r_{1} \leq q+1$ which follows from $r_{1} \leq r \leq q+1$.

Corollary 18. There exists an absolute constant $C>0$ such that $w / q \leq f(w) \leq$ $w / q+C\left(w^{3 / 2} / q^{5 / 2}+1\right)$ for all even $w$ with $C q^{3 / 2} \leq w \leq N-C q^{3 / 2}$.

Note that for odd $w, N-w$ is even and so $(N-w) / q \leq f(w)=f(N-w) \leq$ $(N-w) / q+C\left((N-w)^{3 / 2} / q^{5 / 2}+1\right)$.

Proof. By choosing $C$ sufficiently large we may assume that $q$ is also large. The lower bound follows from Lemmas 12 and 3. For the upper bound choose $r$ minimal such that $r>w / q+2 w^{3 / 2} / q^{5 / 2}$ and $r \equiv q w \bmod 4$. Write $w=r q-4 t$, so that $r^{3 / 2} \leq 4 t \ll r^{2}$ and $r>\sqrt{q}$. By Lemma 16 there exists a simple decomposition of $K_{r}$ with $M(\Pi)=r / 2+2 t$ and indeed, this decomposition must have maximal clique size $r_{1}=r-O(\sqrt{r})$. Then by Lemma 17 this decomposition can be realised by a subset $S$ of $P G(2, q)$. Now $\left|\mathcal{L}^{o}(S)\right|=q r-4 t=w$ by Lemma 14 and so $f(w) \leq r \leq w / q+C\left(w^{3 / 2} / q^{5 / 2}+1\right)$.

\section{Further constructions from blocking sets and the maximum of $f(r)$}

We shall now provide some constructions that give at least some reasonable bounds on $f(r)$ for $r<C q^{3 / 2}$ or $r>N-C q^{3 / 2}$.

Let $Q^{+} \subseteq \mathbb{F}_{q}$ be the set of non-zero quadratic residues and $Q^{-} \subseteq \mathbb{F}_{q}$ be the set of quadratic non-residues. Both sets have $(q-1) / 2$ elements. Define $Q_{i} \subseteq \mathcal{P}, i=0,1$ by

$$
Q_{0}=\left\{[x: 0: 1]: x \in Q^{+}\right\} \cup\left\{[1: x: 0]: x \in Q^{+}\right\} \cup\left\{[0: 1: x]: x \in Q^{-}\right\},
$$

and

$$
Q_{1}=\left\{[x: 0: 1]: x \in Q^{+}\right\} \cup\left\{[1: x: 0]: x \in Q^{+}\right\} \cup\left\{[0: 1: x]: x \in Q^{+}\right\} .
$$

Given any line $\ell: \alpha X+\beta Y+\gamma Z=0$ that does not go through the points $O_{x}:=[1: 0: 0]$, $O_{y}:=[0: 1: 0], O_{z}:=[0: 0: 1]$, we have $\left|\ell \cap Q_{i}\right| \equiv i \bmod 2$. Indeed, $\ell$ intersects $\{[x: 0: 1]$ : $\left.x \in Q^{+}\right\}$iff $\alpha / \gamma \in Q^{+}$and similarly for the others. But for any $\alpha, \beta, \gamma \neq 0$ an odd number of the conditions $\alpha / \gamma \in Q^{+}, \beta / \gamma \in Q^{+}$, and $\gamma / \alpha \in Q^{+}$hold.

The example $Q_{0}$ is due to J. di Paola. By a famous result of Blokhuis [4] the set $Q_{0} \cup\left\{O_{x}, O_{y}, O_{z}\right\}$ is the smallest nontrivial blocking set on $P G(2, q)$ when $q$ is prime.

\section{Lemma 19.}

$$
f\left(\frac{3}{2}(q-1)+k q+j\right) \leq 3 q+j(q+2-j)
$$

for $0 \leq k \leq(q-1) / 2$ and $0 \leq j \leq q+1$.

Proof. Let $V$ be the set of $k q$ points that lie in one of $k$ "vertical" lines of the form $X=\alpha Z, \alpha \in Q^{-}$, not including the point $O_{y}$ at infinity. Let $C$ be any set of $j$ points on the conic $X Z=Y^{2}$. Note that $V, Q_{i}$, and $C$ are pairwise disjoint for $i=0,1$. 


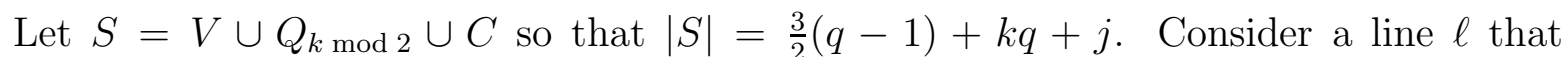
does not meet $\left\{O_{x}, O_{y}, O_{z}\right\}$. Then $|\ell \cap V|=k$ and $\left|\ell \cap Q_{k \bmod 2}\right| \equiv k \bmod 2$. Thus $|\ell \cap S| \equiv|\ell \cap C| \bmod 2$. From the proof of Theorem 4 there are $j(q+2-j)$ lines that meet $C$ in an odd number of points, and there are only $3 q$ lines that meet $\left\{O_{x}, O_{y}, O_{z}\right\}$, so $f(|S|) \leq\left|\mathcal{L}^{o}(S)\right| \leq 3 q+j(q+2-j)$ as required.

\section{Lemma 20.}

$$
f(k q+j) \leq k+j(q+2-j)
$$

for $0 \leq k \leq(q-1) / 2, k$ even, and $0 \leq j \leq q+1$.

Proof. Let $V$ and $C$ be as in the proof of Lemma 19. Then the number of lines meeting $C$ in an odd number of points is $j(q+2-j)$ while the number of lines meeting $V$ in an odd number of points is just $k$ (the lines of $V$ ). As $|V \cup C|=k q+j, f(k q+j) \leq$ $k+j(q+2-j)$.

\section{Lemma 21.}

$$
f(q+1+k q+j) \leq q+1+k+j(q+2-j)
$$

for $0 \leq k \leq(q-1) / 2, k$ even, and $0 \leq j \leq q-1$,

Proof. Let $V$ and $C$ be as in the proof of Lemma 19] except that we shall now insist that $O_{x}, O_{z} \notin C$. Let $C^{\prime}$ be the conic $X Z=4 Y^{2}$. Note that $C^{\prime}$ could only meet $C$ at the points $O_{x}, O_{z}$, which we have assumed do not lie in $C$. Also $C^{\prime} \cap V=\emptyset$. There are $q+1$ lines that meet $C^{\prime}$ in an odd number of points, $j(q+2-j)$ lines that meet $C$ in an odd number of points, and $k$ lines that meet $V$ in an odd number of points. The result follows since $\left|V \cup C \cup C^{\prime}\right|=q+1+k q+j$.

Corollary 22. For large $q$, the maximum value of $f(r)$ is $\left(q^{2}+4 q+3\right) / 4$ and occurs only at $r=(q+1) / 2, r=(q+3) / 2, r=N-(q+1) / 2$, and $r=N-(q+3) / 2$.

Proof. The result follows when $r$ is restricted to the range $0 \leq r \leq q+1$ and $N-(q+1) \leq$ $r \leq N$ by Theorem 4 and Lemma 2 , so it is enough by Lemma 2 to bound $f(r)$ in the range $r \in[q+2, N / 2]$. For $r \in\left[q+2,\left(\frac{3}{2}-\varepsilon\right) q\right]$ we can apply Lemma 21 with $k=0$ to obtain $f(r) \leq\left(\frac{1}{4}-\varepsilon^{2}\right) q^{2}+O(q)$. For $r \in\left[\left(\frac{3}{2}-\varepsilon\right) q, \frac{3}{2}(q-1)\right]$ we can apply Lemma 19 with $k=j=0$ and Theorem 5 to obtain $f(r) \leq 3 q+(q-1) \varepsilon q$. Thus we may assume $r \geq \frac{3}{2}(q-1)$.

If $|r / q-t| \geq \frac{1}{4}$ for every integer $t$, then we write $r=\frac{3}{2}(q-1)+k q+j$, where either $0 \leq j \leq \frac{3}{2}+\frac{q}{4}$ or $\frac{3}{2}+\frac{3 q}{4} \leq j<q$. In either case Lemma 19 implies

$$
f(r) \leq 3 q+\frac{q+5}{4} \cdot \frac{3 q+3}{4}=\frac{1}{16}\left(3 q^{2}+66 q+15\right) .
$$

If $|r / q-t|<\frac{1}{4}$ and $\lfloor(r-1) / q\rfloor$ is even, we write $r=k q+j$ with $1 \leq j<\frac{q}{4}$ or $\frac{3 q}{4}<j \leq q$. In either case Lemma 20 gives

$$
f(r) \leq k+\frac{3 q+1}{4} \cdot \frac{q+7}{4} \leq \frac{1}{16}\left(3 q^{2}+30 q-1\right) .
$$

Finally, if $|r / q-t|<\frac{1}{4}$ and $\lfloor(r-1) / q\rfloor$ is odd, we write $r=q+1+k q+j$ with $0 \leq j<\frac{q}{4}-1$ or $\frac{3 q}{4}-1<j \leq q$. In either case Lemma 21 gives

$$
f(r) \leq q+1+k+\frac{3 q-3}{4} \cdot \frac{q+11}{4} \leq \frac{1}{16}\left(3 q^{2}+38 q+24\right) .
$$


Thus in all cases

$$
f(r) \leq \frac{1}{16}\left(3 q^{2}+66 q+15\right)<\frac{1}{4}\left(q^{2}+4 q+3\right) .
$$

for $q$ sufficiently large.

\section{Exact values from the Baer subplane}

A subset of points $S \subseteq \mathcal{P}$ is a subplane of order $k$ if $|S|=k^{2}+k+1$ and the sets $\{\ell \cap S: \ell \in \mathcal{L},|\ell \cap S|>1\}$ form the line system of a finite projective plane of order $k$. In the case when $k=\sqrt{q}$, we call $S$ a Baer subplane. It is well known that such Baer subplanes exists whenever $q$ is a perfect square (see Bruck [7]). Even more (see, e.g., Yff [16]) $\mathcal{P}$ can be partitioned into $q-\sqrt{q}+1$ Baer subplanes.

Consider a Baer sublane $B$ and let $R_{B} \subseteq \mathcal{L}$ be the set of lines meeting it in exactly $\sqrt{q}+1$ points. Then $\left|R_{B}\right|=q+\sqrt{q}+1$. The lines of $R_{B}$ cover every point of $B$ exactly $\sqrt{q}+1$ times, and every other point exactly once. Thus $\mathcal{P}^{o}\left(R_{B}\right)=\mathcal{P} \backslash B$, which is very large. However, consider an arbitrary point $p \notin B$ and let $R$ be the symmetric difference of $R_{B}$ and $\mathcal{L}(\{p\})$ (these two families contain only one common line $\ell_{p} \in R_{B}$ through $p$ ). Then $\mathcal{P}^{o}(R)=B \cup\{p\}$. We obtain

$$
f(2 q+\sqrt{q}) \leq q+\sqrt{q}+2 .
$$

Considering $p \in B$ and the set of even lines of $B \backslash\{p\}$ (it is again the symmetric difference of $R_{B}$ and $\mathcal{L}(\{p\})$, now they have $\sqrt{q}+1$ common lines) we obtain

$$
f(2 q-\sqrt{q}) \leq q+\sqrt{q} .
$$

Considering two disjoint Baer subplanes we get

$$
f(2 q+2 \sqrt{q}+2) \leq 2 q+2 \sqrt{q}+2 .
$$

Theorem 23. Equality holds in (7) and (8) for $q \geq 81$.

We also conjecture that equality holds in (9), too (at least for large enough $q$ ). For the proof of Theorem 23 we need the following classical results and a few lemmata.

Lemma 24. (Bruen [8], sharpening by Bruen and Thas [9])

Suppose that $S \subseteq \mathcal{P}$ is a nontrivial blocking set (i.e., it meets every line but does not contain any) then $|S| \geq q+\sqrt{q}+1$. Moreover, if $|S|=q+\sqrt{q}+2$, and $q \geq 9$ is of square order, then there exists a point $x \in S$ such that $S \backslash\{x\}$ is the point set of a Baer subplane.

Let $\mathcal{U} \subseteq \mathcal{L}$ be a set of lines. A set $C \subseteq \mathcal{P}$ is called a near-blocker of $\mathcal{U}$ if it meets exactly all but one member of $\mathcal{U}$.

Lemma 25. Let $\mathcal{U}$ be a set of lines in $P G(2, q)$.

(a) Suppose that $\cap_{\ell \in \mathcal{U}} \ell=\emptyset$. Then there exists a near-blocker of size at most $|\mathcal{U}| / 2$.

(b) Suppose that $q \geq 5$ is odd and $\mathcal{U}$ cannot be blocked by a 2-element set. Then there exists a near-blocker of size at most $|\mathcal{U}| / 3+(q+1) / 6$. 
Proof. (a) Let us apply induction on the size of $|\mathcal{U}|$. The cases $|\mathcal{U}|=1,2,3$ are trivial. If $\mathcal{U}$ cannot be covered by two points then select any point $p \in \mathcal{P}$ covered at least twice by the lines of $\mathcal{U}$ and use induction from $\mathcal{U} \backslash \mathcal{L}(\{p\})$. Otherwise, some two points $x_{1}, x_{2}$ cover all lines. Assuming that $\operatorname{deg}_{\mathcal{U}}\left(x_{1}\right) \geq \operatorname{deg}_{\mathcal{U}}\left(x_{2}\right)$, select $x_{1}$ and one element from all but one of the lines of $\mathcal{U}$ going through $x_{2}$ and avoiding $x_{1}$.

(b) For $|\mathcal{U}| \leq q+2$ we have $\lfloor|\mathcal{U}| / 2\rfloor \leq|\mathcal{U}| / 3+(q+1) / 6$ and we can apply case (a). (If $|\mathcal{U}|=q+2$ we make use of the fact that $q$ is odd.) We may now suppose $|\mathcal{U}| \geq q+3$, so $\max _{p} \operatorname{deg}_{\mathcal{U}}(p) \geq 3$. Consider first the case when $\mathcal{U}$ cannot be covered by three vertices. Chose a maximum degree vertex $p$ and apply the induction hypothesis to $\mathcal{U} \backslash \mathcal{L}(\{p\})$. Finally, if some set $\left\{x_{1}, x_{2}, x_{3}\right\}$ meets every member of $\mathcal{U}$ we choose the two highest degree vertices among them and one element from all but one of the lines of $\mathcal{U}$ going through the third, avoiding the other two. In this way we obtain a near-cover of size at most $2+(|\mathcal{U}| / 3-1)$.

The following lemma will be useful when $\left|\mathcal{L}^{e}(A)\right|, t_{1}$, and $t_{2}$ are all small.

\section{Lemma 26.}

(a) Let $A=\left(\ell \backslash T_{1}\right) \cup T_{2}$ where $\ell$ is a line, $T_{1} \subseteq \ell, T_{2} \cap \ell=\emptyset$, and $t_{i}=\left|T_{i}\right|$. Then $\left|\mathcal{L}^{e}(A)\right| \geq\left(t_{1}+t_{2}\right) q-t_{2}\left(2 t_{1}+t_{2}-2\right)$.

(b) Let $A=\left(B \backslash T_{1}\right) \cup T_{2}$ where $B$ is a Baer subplane, $T_{1} \subseteq B, T_{2} \cap B=\emptyset$, and $t_{i}=\left|T_{i}\right|$. Then $\left|\mathcal{L}^{e}(A)\right| \geq\left(t_{1}+t_{2}\right) q-t_{2}\left(2 t_{1}+t_{2}-1\right)-t_{1} \sqrt{q}$.

Proof. (a) Consider the lines through a point $x \in T_{2}$. Exactly $q+1-t_{1}$ of them meet $\ell \backslash T_{1}$. At most $t_{2}-1$ of these lines contain a further point of $A$ (namely a point from $T_{2}$ ). Thus we have obtained at least $t_{2}\left(q+1-t_{1}-\left(t_{2}-1\right)\right) 2$-point lines. Next consider the $q$ lines through a point $y \in T_{1}$ other than $\ell$. All but $t_{2}$ avoids $T_{2}$, too, thus giving at least $t_{1}\left(q-t_{2}\right)$ zero-point lines. The total number of these lines gives the desired lower bound.

(b) Every point $x \in T_{2}$ is incident to at least $\left(q-t_{1}\right)-\left(t_{2}-1\right) 2$-point lines, and every point $y \in T_{1}$ is incident to at least $q-\sqrt{q}-t_{2}$ zero-point lines.

Proof of equality in (7). Suppose, on the contrary, that we have a set of lines $R,|R|=$ $2 q+\sqrt{q}$, such that for $S=\sum_{\ell \in R} \ell$ we have $|S|<q+\sqrt{q}+2$. Since $|S|$ is even, we have $|S| \leq q+\sqrt{q}$. Since $R$ is odd we have $R=\mathcal{L}^{e}(S)$. Thus $S$ meets every line from $\mathcal{L} \backslash R$. Let $\mathcal{U}$ be the set of lines avoiding $S$, we have $\mathcal{U} \subseteq R$.

First consider the case when there is a set $V,|V| \leq 2$, meeting all points of $\mathcal{U}$. (This includes the case $\mathcal{U}=\emptyset$.) Then $S \cup V$ meets all lines, so is a blocking set.

We claim that $S \cup V$ does not contain a line, so is a non-trivial blocking set. Suppose, on the contrary, that there is a line $\ell \subseteq S \cup V$. Apply Lemma [26 (a) with $A=S=$ $\left(\ell \backslash T_{1}\right) \cup T_{2}$ where $T_{1}=\ell \cap V,\left|T_{1}\right| \leq 2$ and $T_{2}=S \backslash \ell,\left|T_{2}\right| \leq|S \cup V|-|\ell| \leq \sqrt{q}+1$. We obtain that

$$
\left|\mathcal{L}^{e}(S)\right| \geq t_{1} q+t_{2}\left(q+2-2 t_{1}-t_{2}\right) \geq t_{1} q+t_{2}(q-\sqrt{q}-3) .
$$

Since $\left|\mathcal{L}^{e}(S)\right|=2 q+\sqrt{q}$ we obtain that $\left|T_{1}\right|+\left|T_{2}\right| \leq 2$ for $q \geq 49$.

We finish the proof of our claim by observing that for $\left|T_{1}\right|+\left|T_{2}\right| \leq 2, T_{1} \subseteq \ell$, the number of even lines $\left|\mathcal{L}^{e}\left(\left(\ell \backslash T_{1}\right) \cup T_{2}\right)\right|$ cannot be $2 q+\sqrt{q}$. Indeed, in the case $T_{1}=\emptyset$ we 
have $\left|\mathcal{L}^{e}(S)\right| \leq t_{2} q+2<2 q+\sqrt{q}$. In the case $t_{2}=0$ we have $\left|\mathcal{L}^{e}(S)\right| \leq 1+t_{1} q<2 q+\sqrt{q}$. Finally, in the case $t_{1}=t_{2}=1$ we have $\left|\mathcal{L}^{e}(S)\right|=2 q-1<2 q+\sqrt{q}$.

Consider $S \cup V$, which is a non-trivial blocking set of size at most $q+\sqrt{q}+2$. By the Bruen-Thas theorem (Lemma 24) there is a Baer subplain $B \subseteq S \cup V$. Thus we know a lot about the structure of $S$, we can write $S=\left(B \backslash T_{1}\right) \cup T_{2}$ where $T_{1}=B \backslash S$ (it is a subset of $V$, so $\left.t_{1} \leq 2\right)$ and $T_{2}=S \backslash B \subseteq(S \cup V) \backslash B$ so $t_{2} \leq 1$.

We finish the proof of the case $|V| \leq 2$ by checking all possible values of $t_{1}$ and $t_{2}$. In case of $t_{1}=2, t_{2}=1$, Lemma 26 (b) applied to $A=S$ gives $\left|\mathcal{L}^{e}(S)\right| \geq 3 q-4-2 \sqrt{q}$. This exceeds $2 q+\sqrt{q}$ for $q \geq 25$. We obtain that $t_{1}+t_{2} \leq 2$. Since $|S|$ is even and $|B|$ is odd their symmetric difference (i.e., $T_{1} \cup T_{2}$ ) is odd, we get $t_{1}+t_{2}=1$. So $S$ should be one of the examples discussed in the beginning of this section and we are done.

From now on suppose that there is no set $V,|V| \leq 2$, meeting all points of $\mathcal{U}$. Apply Lemma 25 (b) to $\mathcal{U}$ to obtain a near-blocker $C$ of $\mathcal{U}$ of size at most $|\mathcal{U}| / 3+(q+1) / 6$ and a line $\ell_{C} \in \mathcal{U}$ missed by $C$. We proceed as in the proof of Theorem 6 .

The set $S \cup C$ meets all lines except $\ell_{C}$, so it is a blocking set of the affine plane $P G(2, q) \backslash \ell_{C}$. Then Lemma 8 yields $|S \cup C| \geq 2 q-1$. We obtain

$$
2 q-1 \leq|S|+|C| \leq(q+\sqrt{q})+|\mathcal{U}| / 3+(q+1) / 6 .
$$

Here $|\mathcal{U}| \leq|R|=2 q+\sqrt{q}$ so the right hand side is at most $(11 q+8 \sqrt{q}+1) / 6$. This cannot hold for $q \geq 81$. This final contradiction implies that $|S| \leq q+\sqrt{q}$ is not possible for $q \geq 81$ and we are done.

Proof of equality in (8) . This proof is similar to the previous proof, but simpler. Suppose, on the contrary, that we have a set of lines $R,|R|=2 q-\sqrt{q}$ such that for $S=\sum_{\ell \in R} \ell$ we have $|S|<q+\sqrt{q}$. As $|S|$ is even, we have $|S| \leq q+\sqrt{q}-2$. Since $R$ is odd we have $R=\mathcal{L}^{e}(S)$. Thus $S$ meets every line from $\mathcal{L} \backslash R$. Let $\mathcal{U}$ be the set of lines avoiding $S$, so that $\mathcal{U} \subseteq R$.

If there is a set $V,|V| \leq 2$, meeting all points of $\mathcal{U}$ (including the case $\mathcal{U}=\emptyset$ ) then $S \cup V$ meets all lines, it is a blocking set of size at most $q+\sqrt{q}$. By the Bruen theorem (Lemma 24) it must contain a line $\ell$. Apply Lemma 26 (a) with $A=S=\left(\ell \backslash T_{1}\right) \cup T_{2}$ where $T_{1}=\ell \cap V,\left|T_{1}\right| \leq 2$ and $T_{2}=S \backslash \ell,\left|T_{2}\right| \leq|S \cup V|-|\ell| \leq \sqrt{q}-1$. We obtain that

$$
\left|\mathcal{L}^{e}(S)\right| \geq t_{1} q+t_{2}\left(q+2-2 t_{1}-t_{2}\right) \geq t_{1} q+t_{2}(q-\sqrt{q}-1) .
$$

Since $\left|\mathcal{L}^{e}(S)\right|=2 q-\sqrt{q}$ we obtain that $\left|T_{1}\right|+\left|T_{2}\right| \leq 2$ for $q \geq 25$.

We finish the investigation of this case by observing that for $\left|T_{1}\right|+\left|T_{2}\right| \leq 2, T_{1} \subseteq \ell$, the number of even lines $\left|\mathcal{L}^{e}\left(\left(\ell \backslash T_{1}\right) \cup T_{2}\right)\right|$ cannot be $2 q-\sqrt{q}$. Since both $S$ and $\ell$ are even sets, their symmetric difference (i.e., $T_{1} \cup T_{2}$ ) is even. We have four cases to check according to the value of $\left(t_{1}, t_{2}\right) \in\{(2,0),(1,1),(0,2),(0,0)\}$. The sizes of $\left|\mathcal{L}^{e}(S)\right|$ are $2 q+1,2 q-1$, again $2 q+1$, and 1 , respectively. None of these is equal to $2 q-\sqrt{q}$.

From now on suppose that $\mathcal{U} \neq \emptyset$ and there is no set $V,|V| \leq 2$, meeting all points of $\mathcal{U}$. Apply Lemma 25 (b) to $\mathcal{U}$ to obtain a near-blocker $C$ of $\mathcal{U}$ of size at most $|\mathcal{U}| / 3+(q+1) / 6$ and a line $\ell_{C} \in \mathcal{U}$ missed by $C$. We proceed as in the proof of Theorem 6.

The set $S \cup C$ meets all lines except $\ell_{C}$, so it can be considered as a blocking set of the affine plane $P G(2, q) \backslash \ell_{C}$. Then Lemma 8 yields $|S \cup C| \geq 2 q-1$. We obtain

$$
2 q-1 \leq|S|+|C| \leq(q+\sqrt{q}-2)+|\mathcal{U}| / 3+(q+1) / 6 .
$$


Here $|\mathcal{U}| \leq|R|=2 q-\sqrt{q}$ so the right-hand-side is at most $(11 q+4 \sqrt{q}-11) / 6$. This cannot hold for $q \geq 49$ implying that $|S| \leq q+\sqrt{q}$ is not possible for $q \geq 49$ and we are done.

With some more work we can see that only the examples from the Baer subplane give equalities in (7) and (8) (for $\left.q>q_{0}\right)$.

Many questions remain open. What is $f(q+2)$, and $f(q+3)$ ? The least we should be able to do is to prove better bounds on these. Also, any information about $f(r)$ for $r \leq 2 q^{3 / 2}$ would be great.

\section{Acknowledgements}

The authors are indebted to the referees for their helpful comments and suggestions. 


\section{References}

[1] Alon N., Combinatorial Nullstellensatz, Combin. Prob. Comput. 8 (1999), 7-29.

[2] Bichara A. and Korchmáros G., Note on $(q+2)$-sets in a Galois plane of order q. Combinatorial and geometric structures and their applications (Trento, 1980), pp. 117-121, Ann. Discrete Math., 14, North-Holland, Amsterdam-New York, 1982.

[3] Besicovitch S., The Kakeya problem. Amer. Math. Monthly 70 (1963), 697-706.

[4] Blokhuis A., On the size of a blocking set in $P G(2, p)$. Combinatorica 14 (1994), 111-114.

[5] Blokhuis A. and Mazzocca F., The finite field Kakeya problem. Building bridges, 205-218, Bolyai Soc. Math. Stud., 19, Springer, Berlin, 2008.

[6] Brouwer A. E. and Schrijver A., The blocking number of an affine space. J. Combin. Theory Ser. A 24 (1978), 251-253.

[7] Bruck R. H., Quadratic extensions of cyclic planes. Proc. Sympos. Appl. Math., Vol. 10 pp. 15-44. American Mathematical Society, Providence, R.I. 1960.

[8] Bruen A., Blocking sets in finite projective planes. SIAM J. Appl. Math. 21 (1971), 380-392.

[9] Bruen A. A. and Thas J. A., Blocking sets. Geometriae Dedicata 6 (1977), 193-203.

[10] Faber X. W. C., On the finite field Kakeya problem in two dimensions. J. Number Theory 124 (2007), 248-257.

[11] Hirschfeld J. W. P., Projective geometries over finite fields. Second edition. Oxford Mathematical Monographs. The Clarendon Press, Oxford University Press, New York, 1998. xiv+555 pp.

[12] Jamison R., Covering finite fields with cosets of subspaces. J. Combin. Theory Ser. A 22 (1977), 253-266.

[13] Lovász L. and Schrijver A., Remarks on a theorem of Rédei. Studia Scient. Math. Hungar. 16 (1981), 449-454.

[14] Ryser H. J., Geometries and incidence matrices. Amer. Math. Monthly 62 (1955), 25-31.

[15] Szőnyi T., Blocking sets in Desarguesian affine and projective planes. Finite Fields Appl. 3 (1997), 187-202.

[16] Yff P., On subplane partitions of a finite projective plane. J. Combinatorial Theory Ser. A 22 (1977), 118-122. 


\section{Appendix A. Values of $f(r)$ for small $q$.}

Table 1: $q=3$

\begin{tabular}{llll}
$r$ & $f(r)$ & $r$ & $f(r)$ \\
\hline 1 & 4 & 4 & 4 \\
2 & 6 & 5 & 4 \\
3 & 6 & 6 & 2
\end{tabular}

Table 2: $q=5$

\begin{tabular}{rlrlrl}
$r$ & $f(r)$ & $r$ & $f(r)$ & $r$ & $f(r)$ \\
\hline 1 & 6 & 6 & 6 & 11 & 4 \\
2 & 10 & 7 & 8 & 12 & 4 \\
3 & 12 & 8 & 8 & 13 & 6 \\
4 & 12 & 9 & 6 & 14 & 6 \\
5 & 10 & 10 & 2 & 15 & 4
\end{tabular}

Table 3: $q=7$

\begin{tabular}{rlrlrlrl}
$r$ & $f(r)$ & $r$ & $f(r)$ & $r$ & $f(r)$ & $r$ & $f(r)$ \\
\hline 1 & 8 & 8 & 8 & 15 & 6 & 22 & 6 \\
2 & 14 & 9 & 12 & 16 & 8 & 23 & 6 \\
3 & 18 & 10 & 10 & 17 & 8 & 24 & 4 \\
4 & 20 & 11 & 10 & 18 & 6 & 25 & 8 \\
5 & 20 & 12 & 12 & 19 & 10 & 26 & 6 \\
6 & 18 & 13 & 8 & 20 & 4 & 27 & 6 \\
7 & 14 & 14 & 2 & 21 & 8 & 28 & 4
\end{tabular}


Table 4: $q=9$

\begin{tabular}{llrlrlrlll}
$r$ & $f(r)$ & $r$ & $f(r)$ & $r$ & $f(r)$ & $r$ & $f(r)$ & & \\
\hline 1 & 10 & 10 & 10 & 19 & 8 & 28 & 4 & 37 & 6 \\
2 & 18 & 11 & 16 & 20 & 12 & 29 & 10 & 38 & 6 \\
3 & 24 & 12 & 12 & 21 & 10 & 30 & 6 & 39 & 8 \\
4 & 28 & 13 & 14 & 22 & 10 & 31 & 8 & 40 & 8 \\
5 & 30 & 14 & 14 & 23 & 12 & 32 & 4 & 41 & 10 \\
6 & 30 & 15 & 12 & 24 & 8 & 33 & 10 & 42 & 6 \\
7 & 28 & 16 & 16 & 25 & 10 & 34 & 6 & 43 & 8 \\
8 & 24 & 17 & 10 & 26 & 10 & 35 & 8 & 44 & 8 \\
9 & 18 & 18 & 2 & 27 & 12 & 36 & 4 & 45 & 6
\end{tabular}

Table 5: $q=11$

\begin{tabular}{rlrlrlrlrlll}
$r$ & $f(r)$ & $r$ & $f(r)$ & $r$ & $f(r)$ & $r$ & $f(r)$ & $r$ & $f(r)$ & $r$ & $f(r)$ \\
\hline 1 & 12 & 12 & 12 & 23 & 10 & 34 & 10 & 45 & 8 & 56 & 8 \\
2 & 22 & 13 & 20 & 24 & 16 & 35 & 14 & 46 & 6 & 57 & 8 \\
3 & 30 & 14 & $14-26$ & 25 & 16 & 36 & 4 & 47 & 10 & 58 & 6 \\
4 & 36 & 15 & $14-18$ & 26 & 14 & 37 & 12 & 48 & 8 & 59 & 10 \\
5 & 40 & 16 & 16 & 27 & 14 & 38 & 10 & 49 & 12 & 60 & 8 \\
6 & 42 & 17 & 16 & 28 & 12 & 39 & 10 & 50 & 6 & 61 & 8 \\
7 & 42 & 18 & $14-18$ & 29 & 16 & 40 & 4 & 51 & 10 & 62 & 10 \\
8 & 40 & 19 & $14-26$ & 30 & 10 & 41 & 12 & 52 & 8 & 63 & 10 \\
9 & 36 & 20 & $16-20$ & 31 & $14-18$ & 42 & 6 & 53 & 12 & 64 & 8 \\
10 & 30 & 21 & 12 & 32 & 12 & 43 & 14 & 54 & 6 & 65 & 8 \\
11 & 22 & 22 & 2 & 33 & 16 & 44 & 4 & 55 & 10 & 66 & 6
\end{tabular}

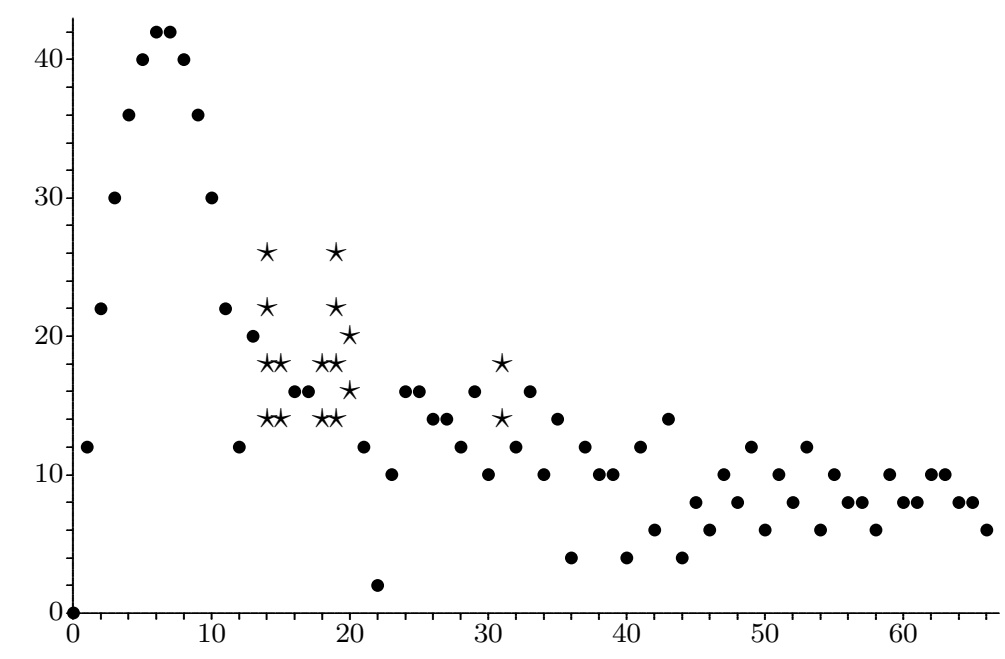

Figure 1: Graph of $f(r)$ for $q=11$. Dots represent known values, and stars represent possible values for the values of $r$ for which $f(r)$ is unknown. 